\title{
Intervención Educativa de Enfermería en las Capacidades de Autocuidado de personas con Diabetes Tipo 2 de Tenosique, Tabasco
}

\author{
Jorge Eduardo Méndez Valdéz \\ Egresado de la Licenciatura en Enfermería, División Académica \\ Multidisciplinaria de los Ríos, Universidad Juárez Autónoma de Tabasco, \\ México
}

Armando Miranda de la Cruz

Profesores Investigadores de la División Académica Multidisciplinaria de los Ríos, Universidad Juárez Autónoma de Tabasco, México

\section{Elia del Carmen Martínez Ruíz \\ Esther Alice Jiménez Zuñiga}

Profesores Investigadores de la Facultad de Enfermería de la Universidad

Veracruzana, México

Janett Marina García Hernández.

Jairo David Contrera Madrigal

Profesores Investigadores de la División Académica Multidisciplinaria de los

Ríos, Universidad Juárez Autónoma de Tabasco, México

Doi: 10.19044/esj.2018.v14n24p379 URL:http://dx.doi.org/10.19044/esj.2018.v14n24p379

\begin{abstract}
One of these diseases with major social, economic and epidemiological impact is the Diabetes Mellitus (DM), which according to the WHO (2013) is characterized by chronic hyperglycemia and disorders of the metabolism of the carbohydrates, the fats and the proteins as consequence of anomalies of the secretion or of the effect of the insulin. This investigation is sustained in the General Theory of the Deficit of Autocare Dorothea Orem's (TGDA). The aim was to determine the effect of an educational intervention of infirmary in the Capacities of Autocare (CAC) and the State of health (BELONGS) to the persons with DM2. The design is quasi-experimental of longitudinal court, with one $n=30$ participants, I divide in 2 groups [Group Control (GC) and Experimental Group (GE)]. It was in use like I orchestrate the "Scale to estimate Capacities of Autocare ". There was designed an Educational Intervention of Infirmary (IEE) by 12 meetings. The results showed increase in the CAC with an average of 39.33 (GIVE 2.60) in the pretest and 82.20 (GIVE 2.90) in the pos test, the CAC present negative significant relation with
\end{abstract}


the IMC [rs =-.330 ( $\mathrm{p}<0.01)]$, capillary blood sugar $[\mathrm{rs}=-.542(\mathrm{p}<0.01)]$, cholesterol [rs $=-.367(\mathrm{p}<0.01)]$, triglycerides $[\mathrm{rs}=-.587(\mathrm{p}<0.01)]$ and hemoglobin glucosilada $[\mathrm{rs}=-.478(\mathrm{p}<0.01)]$. On having realized the test $\mathrm{T}$ de Student for related samples obtained one $\mathrm{t}=-61.588$ with 14 degrees of freedom for a confidence interval of $95 \%$, locating an average of -42.86667 ; what shows that it exists a difference of averages enters the pre and pos tries by means of a positive effect of the IEE in the CAC.

Keywords: Health Status, Diabetes Mellitus, Index of corporal mass.

\section{Resumen}

Una de estas enfermedades con mayor impacto social, económico y epidemiológico es la Diabetes Mellitus (DM), la cual según la OMS (2013) se caracteriza por hiperglucemia crónica y trastornos del metabolismo de los carbohidratos, las grasas y las proteínas como consecuencia de anomalías de la secreción o del efecto de la insulina. Esta investigación está sustentada en la Teoría General del Déficit de Autocuidado (TGDA) de Dorothea Orem. El objetivo fue determinar el efecto de una intervención educativa de enfermería en las Capacidades de Autocuidado (CAC) y el Estado de Salud (ES) de las personas con DM2. El diseño es cuasi-experimental de corte longitudinal, con una $n=30$ participantes, divido en 2 grupos [Grupo Control (GC) y Grupo Experimental (GE)]. Se utilizó como instrumento la "Escala para Estimar Capacidades de Autocuidado". Se diseñó una Intervención Educativa de Enfermería (IEE) con 12 sesiones. Los resultados mostraron aumento en las CAC con una media de 39.33 (DE 2.60) en el pre test y 82.20 (DE 2.90) en el pos test, las CAC presentan relación negativa significativa con el IMC [rs=$.330(\mathrm{p}=0.01)]$, glicemia capilar [ $\mathrm{rs}=-.542(\mathrm{p}=0.01)]$, colesterol $[\mathrm{rs}=-.367$ $(\mathrm{p}=0.01)]$, triglicéridos $[\mathrm{rs}=-.587(\mathrm{p}=0.01)]$ y hemoglobina glucosilada [rs=$.478(\mathrm{p}=0.01)]$. Al realizar la prueba $\mathrm{T}$ de Student para muestras relacionadas se obtuvo una $\mathrm{t}=-61.588$ con 14 grados de libertad para un intervalo de confianza del $95 \%$, ubicando una media de -42.86667 ; lo que muestra que existe una diferencia de medias entra la pre y pos prueba mediante un efecto positivo de la IEE en las CAC.

Palabras claves: Estado de Salud, Diabetes Mellitus, Índice de Masa Corporal.

\section{Introduction}

La diabetes es una grave enfermedad crónica que se desencadena cuando el páncreas no produce suficiente insulina (una hormona que regula el nivel de azúcar, o glucosa, en la sangre), o cuando el organismo no puede utilizar con eficacia la insulina que produce. La diabetes es un importante 
problema de salud pública y una de las cuatro enfermedades no transmisibles (ENT) seleccionadas por los dirigentes mundiales para intervenir con carácter prioritario. En las últimas décadas han aumentado sin pausa el número de casos y la prevalencia de la enfermedad (Organización Mundial de la Salud [OMS], 2016; OMS, 2013; OMS, 2010; Organizacion Panamericana de la Salud [OPS], 2010).

Una de estas enfermedades con mayor impacto social y epidemiológico es la Diabetes Mellitus (DM), la cual según la OMS (2013) es un trastorno metabólico que tiene causas diversas; se caracteriza por hiperglucemia crónica y trastornos del metabolismo de los carbohidratos, las grasas y las proteínas como consecuencia de anomalías de la secreción o del efecto de la insulina; con el tiempo, la enfermedad puede causar daños, disfunción e insuficiencia de diversos órganos.

Según las estimaciones, 422 millones de adultos en todo el mundo tenían diabetes en 2014, frente a los 108 millones de 1980. La prevalencia mundial (normalizada por edades) de la diabetes casi se ha duplicado desde ese año, pues ha pasado del $4.7 \%$ al $8.5 \%$ en la población adulta. Ello supone también un incremento en los factores de riesgo conexos, como el sobrepeso o la obesidad; se calcula que en el 2012 la diabetes provocó 1.5 millones de muertes Un nivel de glucosa en la sangre superior al deseable provocó otros 2.2 millones de muertes, al incrementar los riesgos de enfermedades cardiovasculares y de otro tipo. Un $43 \%$ de estos 3.7 millones de muertes ocurren en personas con menos de 70 años, el porcentaje de muertes atribuibles a una glucemia elevada o la diabetes en menores de 70 años de edad es superior en los países de ingresos bajos y medianos que en los de ingresos altos (OMS, 2016; Instituto Nacional de Estadistica Española [INEE], 2013).

La Encuesta Nacional de Salud y Nutrición de Medio Camino (ENSANUTMC, 2016) encontró que la prevalencia de diabetes en el país pasó de $9.2 \%$ en 2012 a $9.4 \%$ en 2016 , esto en base a un diagnóstico previo de la enfermedad; las mujeres reportan mayores valores de diabetes $(10.3 \%)$ que los hombres (8.4\%). Esta tendencia se observa tanto en localidades urbanas ( $10.5 \%$ en mujeres y $8.2 \%$ en hombres) como en rurales $(9.5 \%$ en mujeres, $8.9 \%$ en hombres). La mayor prevalencia de diabetes se observa entre los hombres de 60 a 69 años $(27.7 \%)$, y las mujeres de este mismo rango de edad (32.7\%) y de 70 a 79 años (29.8\%) (Córdova et. al., 2008).

En el estado de Tabasco, la DM ocupa el primer lugar como causa de mortalidad en mujeres a partir de los 30 años, con una prevalencia del $12.9 \%$ en edades de 30-44 años, 28.3\% en edades de 45 a 59 años y del $22.4 \%$ en edades de 60 años y más; en la población masculina la DM ocupa el primer lugar como causa de mortalidad en personas a partir de los 45 años, con el $19.9 \%$ en edades de 45-59 años y $16.9 \%$ en hombres mayores de 60 años. En 
el 2011 fallecieron 1,671 personas por DM, con una incidencia del 73.3 por cada 100,000 habitantes en población general (hombres 71.4 y mujeres 74.9); de acuerdo a la edad el $69.2 \%$ de los fallecimientos por DM ocurrieron en personas mayores de 60 años. En el 2011 se registraron 8,141 casos nuevos (393.22) por DM, 3,420 en hombres (337.18) y 4721 mujeres (44.05) ocupando el vigésimo lugar a nivel nacional (Instituto Nacional de Estadística y Geografía Informatica [INEGI], 2013).

En el Municipio de Tenosique, ocupa el primer lugar como causa de mortalidad en mujeres con 12 casos (3.98\%) y el 2 do. lugar en hombres con 14 (4.86\%); como causa de morbilidad representa el lugar décimo segundo en hombres con 76 casos (26.38\%) y el décimo tercero en mujeres 119 (39.47\%) hasta septiembre del año pasado; se diagnosticaron 21 personas en el último año de las cuales 11 son mujeres de 20 a 59 años, 9 hombres del 20 a 59 años y 1 es hombre mayor de 60 años (Jurisdicción Sanitaria Tenosique, 2014).

Para efectos de aplicación de esta investigación se tomaron conceptos de la Teoría del Déficit de Autocuidado (TEDA) de Dorothea Orem (Kozier, Berman, Snyder \& Erb, 2013; Alligood \& Marriner, 2011) la cual es considerada como una gran teoría debido a la complejidad de sus conceptos, enunciados teóricos, definiciones, premisas, asociaciones y organización. Orem menciona que esta teoría no ha recibido influencia directa de otra teoría; sin embargo, afirma haber considerado la experiencia de la interpretación de otras teóricas de enfermería, concluye que esta explicación al fenómeno de autocuidado (AC) es resultado de su experiencia personal y la construcción fue realizada en base a sus conocimientos sobre el tema abordado (Herrera, et. al., 2012; Cutcliffe, Mckenna \& Hyrkas, 2011).

Considerando los conceptos de la TAC y TSE propuestas por Dorothea Orem, se plantea para esta investigación y para efectos de la explicación de la relación entre las variables de estudio con la teoría de enfermería una estructura mental retomando los conceptos de la teórica que se utilizaron en esta investigación (Ver Figura 1). 


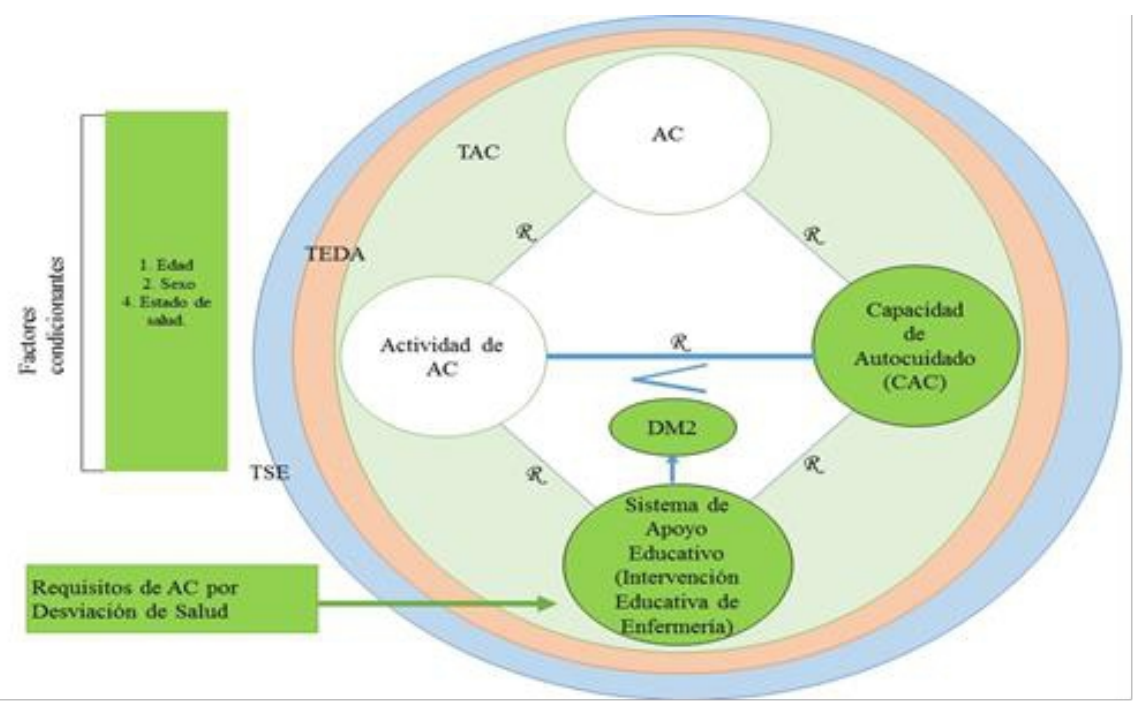

Figura 1. Adaptación del Sistema conceptual de Enfermería. R, relación; <, relación de déficit, actual o prevista. De Orem, D.E. (1995). Nursing Concepts of Practice. San Luis, P. 435.

De acuerdo con el esquema anterior la TEDA está integrada por tres sub-teorías de enfermería; de la TAC se retomaron los conceptos de requisitos de autocuidado por desviación de salud y los factores condicionantes básicos que para efectos de esta investigación fueron: edad, sexo y estado de salud; este último fue medido por variables clínicas, ya que desde el punto de vista de Orem este se usa en el sentido de un estado de la persona caracterizado por el buen estado o integridad de las estructuras humanas desarrolladas y del funcionamiento corporal y mental.

En el modelo de Orem se establece una relación entre la CAC y la Actividad de Enfermería (AE), desde esta perspectiva se retoma de la TSE el Sistema de Apoyo Educativo (SAE) aplicando el método "enseñar" planteando una Intervención Educativa de Enfermería (IEE) que cuya meta final fue incrementar las CAC de autocuidado de la persona y modificar el factor condicionante básico más directo, es decir el ES de las personas con DM2.

En cuanto a la Teoría de los Sistemas Enfermería Dorothea Orem menciona que el sistema de apoyo educativo la enfermera regula el ejercicio y desarrollo de la actividad de autocuidado y la persona cumple con el autocuidado, utilizando la educación y promoción para la salud como elementos primordiales (Orem citada en Alligood \& Marriner, 2014, Págs. 175-187).

De la Clasificacion de Intervenciones de Enfermeria (NIC, 2013) se retoman las intervenciones de enfermería y actividades con el objeto de darles una constitución de acuerdo al método científico aplicado en la ciencia 
enfermera, es decir, el proceso de enfermería (Bulechek, Butcher, Dochterman \& Wagner., 2014).

Para esta investigación se realizó una intervención de enfermería de índole educativa de manera grupal con la finalidad de desarrollar capacidades de autocuidado y mejorar el estado de salud en los pacientes con DM2, esta intervención educativa es denominada "Yo sí puedo cuidarme"; constará de 12 sesiones tomadas de los requisitos de autocuidado por desviación de salud y considerando los factores condicionantes de la Teoría del Autocuidado de Dorothea Orem y con previa revisión de las investigaciones realizadas con antelación en personas con características similares, además considerando aspectos metodológicos de la Clasificación de Intervenciones de Enfermería (Bulechek, Butcher., Dochterman \& Wagner., 2014) (Ver Figura 2).

De acuerdo con lo anterior se planteó la siguiente pregunta de investigación:

¿Cuál es el efecto de una intervención de enfermería en las capacidades de autocuidado y el estado de salud de los pacientes con DM2, que acuden al Hospital Comunitario de Tenosique, Tabasco?

\section{Objetivo}

Determinar el efecto de una intervención de enfermería en las capacidades de autocuidado y el estado de salud de las personas con DM2 que acuden al Hospital Comunitario del municipio de Tenosique, Tabasco.

\section{Hipótesis}

Hi La IEE incrementa las CAC e influye positivamente en el ES de las personas con DM2.

Ho La IEE no incrementa las CAC ni mejora el ES de las personas con DM2. Ha La IEE no incrementa las CAC, pero influye positivamente en el ES de las personas con DM2.

\section{Metodología}

Diseño: cuantitativo, cuasi experimental, longitudinal; ya que en esta investigación se obtuvieron resultados numéricos de variables cuantitativas; se define como cuasi-experimental porque existe un grado de manipulación sobre los sujetos y las variables de investigación (variable dependiente) mediante la intervención educativa en las CAC y el ES de las personas con DM2 que acuden a control y tratamiento al Hospital Comunitario de Tenosique; es un estudio de corte longitudinal debido a que el instrumento y las variables clínicas (estado de salud) se midieron en dos ocasiones un pretest y post-test durante la investigación con una diferencia de tres meses entre cada medición (Pollit \& Hungler, 2000; Hernández Sampieri et. al, 2014). 
Participaron en el estudio 30 personas seleccionadas de acuerdo al muestreo establecido con edades de 40 a 60 años; 15 para el Grupo Control (GC) y 15 personas para el Grupo de Experimental (GE).

\section{Características de los participantes}

- Personas del sexo masculino o femenino que acuden a control y tratamiento de la Diabetes Mellitus tipo II en el Hospital Comunitario de Tenosique.

- Con un mínimo de un mes y un máximo de diez años de diagnósticos que acudan a control mensual en el Núcleo Básico No. 6.

- Personas que no sufran consecuencias graves de la enfermedad como: retinopatía, pie diabético, cetoacidosis diabética, acidosis o alcalosis metabólica.

- Personas que no presenten un estado neurológico con alteraciones o padecimientos psiquiátricos que imposibiliten la comprensión de las temáticas de las sesiones planteadas y que sean dependientes absolutos de un cuidador principal.

\section{Material/instrumentos}

Para efectos de la investigación se utilizaron dos métodos de recolección de datos: el cuestionario auto-administrado para medir las CAC y la medición de variables clínicas (toma de laboratorios) para medir el estado de salud. Se aplicó el instrumento a las personas con DM2 que no están relacionados con el estudio con la finalidad de conocer el abordaje de la intervención educativa de acuerdo a las necesidades identificadas.

El primer método incluye un cuestionario auto-administrado para conocer los aspectos sociodemográficos y las CAC que la persona con DM2 debe desarrollar para lograr el autocuidado, que consta de 2 apartados:

En el segundo apartado se utilizó una Escala para Estimar las Capacidades de Autocuidado (EECAC) este instrumento valora las capacidades de autocuidado representando todos los componentes de poder y ha sido utilizado en población mexicana por Da Silva (1997) y Gallegos (1995), quienes reportaron un Alpha de Cronbach de .79 y $\quad .81$ respectivamente; consta de 24 reactivos con una escala de respuesta tipo Likert con opciones de respuesta que van de nunca $=1$, raras veces $=2$, frecuentemente $=3 \mathrm{y}$ siempre $=4$. La puntuación total del instrumento oscila de 24 a 96 puntos (Contreras O. A., Contreras M. A., Hernández, Castro \& Navarro, 2013). 
Tabla 1. Categorías de las Capacidades de Autocuidado

\begin{tabular}{|c|c|c|c|}
\hline $\begin{array}{l}\text { Nombre de la } \\
\text { variable }\end{array}$ & $\begin{array}{l}\text { No. de } \\
\text { Reactivos }\end{array}$ & Puntaje & Variable Categorizada \\
\hline $\begin{array}{ll}\text { Capacidades } & \text { de } \\
\text { Autocuidado } & \end{array}$ & 24 & $\begin{array}{l}\text { Respuesta: } \\
\text { Nunca (1) } \\
\text { Raras Veces (2) } \\
\text { Frecuentemente (3) } \\
\text { Siempre (4) } \\
\text { Para totalizar: } \\
\text { Mínimo de } 24 \text { puntos. } \\
\text { Máximo de } 96 \text { puntos. }\end{array}$ & $\begin{array}{l}\text { Capacidades } \\
\text { de } 24-47 \text { puntos } \\
\text { Capacidades } \\
\text { 48-71 puntos } \\
\text { Capacidades } \\
\text { Especializadas } \\
\text { puntos }\end{array}$ \\
\hline
\end{tabular}

Para las variables clínicas en el grupo control y experimental se tomaron muestras de laboratorio al inicio y al final de la intervención con ayuno de 12 hrs. En área de laboratorio del Hospital Comunitario de Tenosique; se programó la toma de muestras el mismo día para ambos grupos, calendarizado de acuerdo al inicio y termino de la intervención como laboratorios necesarios para conocer el ES en las personas con DM2; esta variable fue categorizada de acuerdo a lo estipulado en la NOM-015-SSA2-2010 de la siguiente manera:

Tabla 2. Categorías del Estado de Salud

\begin{tabular}{lllll}
\hline $\begin{array}{l}\text { Nombre } \\
\text { de la } \\
\text { variable }\end{array}$ & \multicolumn{1}{c}{ Factores } & Bueno (3) & $\begin{array}{c}\text { Categorías del ES } \\
\text { Regular (2) }\end{array}$ & Malo (1) \\
\hline $\begin{array}{l}\text { Estado de } \\
\text { Salud }\end{array}$ & $\begin{array}{l}\text { Glucemia en ayunas } \\
(\mathrm{mg} / \mathrm{dl})\end{array}$ & $<110$ & $110-140$ & $>140$ \\
& PA sistólica & $<120$ & $121 / 129$ & $>130$ \\
& Colesterol total $(\mathrm{mg} / \mathrm{dl})$ & $<200.0$ & $200-239$ & $\geq 240$ \\
& Triglicéridos en ayuno & $<150$ & $150-200$ & $>200$ \\
& (mg/dl) & $<60 / 80$ & $81-84$ & $>85$ \\
& P.A. diastólica (mm de & & & $>27$ \\
& Hg) & $<25$ & $25-27$ & $>8 \% \mathrm{mg} / \mathrm{dl}$ \\
& Índice de Masa Corporal & $<6.5 \% \mathrm{mg} / \mathrm{dl}$ & $6.5-8 \% \mathrm{mg} / \mathrm{dl}$ & 7 \\
\hline
\end{tabular}

Para las medidas antropométricas se utilizó una báscula con estadiómetro para la toma de peso y talla, el cálculo de índice de masa corporal fue ejecutado a través del paquete estadístico SPSS versión 23.0; para la medición de la tensión arterial se utilizó un baumanómetro con estetoscopio previamente calibrado y para la glicemia capilar un glucómetro marca One Toch.

\section{Procedimiento}

Los datos fueron recolectados en el aula CAPA-UNEME del Hospital Comunitario de Tenosique, en lugar cerrado climatizado, se procuró un ambiente agradable en sesión grupal, para dar respuesta al cuestionario autoadministrado, los instrumentos fueron aplicados en dos ocasiones (pre-test y 
pos-test). El pre-test fue la primera medición del instrumento $\left(\mathrm{O}_{1}\right)$ y pos-test $\left(\mathrm{O}_{2}\right)$ al término de la intervención; se estableció el turno matutino con horario de 10:00 a 11:30 hrs debido a que es un horario accesible para la institución y las personas que asisten a control y tratamiento de la DM2.

\section{Dosificación de la intervención}

La intervención educativa de enfermería consta de 12 sesiones (una semanal) con un tiempo promedio de 120 minutos cada una, estableciendo un total de 1,440 minutos para la intervención; la IEE tiene en su conteniendo los requisitos para el AC por desviación de salud y los FCB de la sub-teoría del AC de Dorothea Orem; así mismo se plantearon de acuerdo a las intervenciones de enfermería del programa de atención a las enfermedades metabólicas legitimado por la NOM-015-SSA2-2010 para la prevención, tratamiento y control de la DM, los factores protectores señalados por la OMS y las investigaciones descritas en el marco empírico (Ver Figura 2), las sesiones planteadas se detallan a continuación (Fernández, 2010; Rodríguez \& Vallenilla, 2007; Ávila, Meza, Frías, Sánchez, Vega \& Hernández, 2006; Lezama, Malavé \& Tovar, 2005).

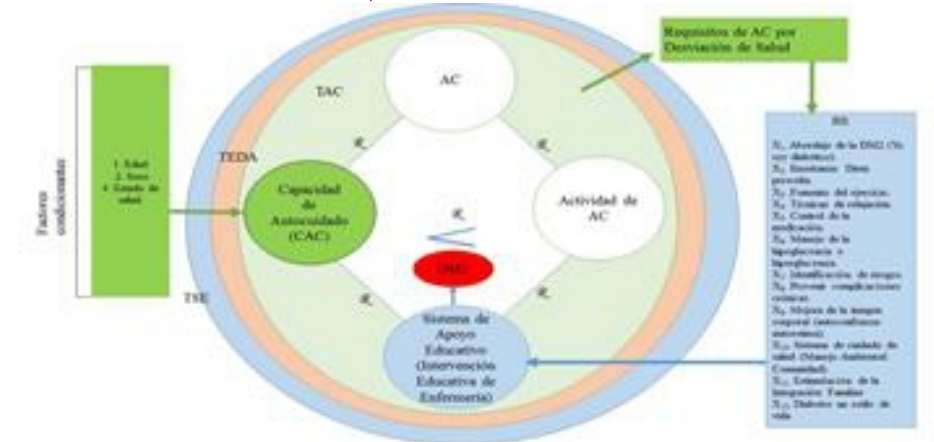

Figura 2. Intervención educativa de Enfermería según TSE (Sistema de Apoyo Educativo) utilizando el método "Enseñar".

\section{Evaluación de la Intervención}

Para efectos de cumplimiento de los objetivos se establece una evaluación cuantitativa de la IEE con la aplicación de un pre-test (O1) y postest $(\mathrm{O} 2)$ cuyas mediciones se realizaron para determinar la eficacia de la intervención educativa de enfermería sobre las capacidades de autocuidado y el estado de salud de los pacientes con DM2 (Ver Figura 2.). Para esta investigación se contempla un análisis inferencial mediante la prueba $\mathrm{T}$ de Student para determinar el efecto de la IEE en las CAC y el ES de las personas con DM2. 


\section{Resultados}

En el siguiente apartado se describen los resultados de la aplicación del instrumento "Escala para Estimar Capacidades de Autocuidado", el cual mostró un $\alpha=0.91$ con un intervalo de confianza del 95\%; este fue aplicado en personas con DM2 que acuden a control y tratamiento en el Hospital Comunitario de Tenosique, Tabasco; a continuación se describen los resultados en función de los objetivos del trabajo.

\section{Variables de estudio}

Para dar cumplimiento al Objetivo Específico No. 1 que menciona la identificación del nivel de CAC de las personas con DM2 que acuden a control y tratamiento en el Hospital Comunitario de Tenosique; las CAC mostraron aumento con una media de 39.33 (DE 2.60) en el pre test y 82.20 (DE 2.90) en el pos test (Ver Tabla 3).

Tabla 3. Estadística descriptiva de las CAC.

\begin{tabular}{lrrrrrrrrr}
\hline \multirow{2}{*}{ Variables } & \multicolumn{2}{c}{$\mathrm{GC} \mathrm{O}_{1}$} & \multicolumn{2}{c}{$\mathrm{GC} \mathrm{O}_{2}$} & \multicolumn{2}{c}{$\mathrm{GE} \mathrm{O}_{1}$} & \multicolumn{2}{c}{$\mathrm{GE} \mathrm{O}_{2}$} \\
\cline { 2 - 10 } & $\overline{\mathrm{X}}$ & $\mathrm{S}$ & $\overline{\mathrm{X}}$ & $\mathrm{S}$ & $\overline{\mathrm{X}}$ & $\mathrm{S}$ & $\overline{\mathrm{X}}$ & $\mathrm{S}$ \\
\hline CAC & 42.60 & 5.1658 & 53.13 & 5.1111 & 39.33 & 2.6095 & 82.20 & 2.9081 \\
\hline${ }^{*}$ CAC: Capacidades de Autocuidado. & & & & & & & & &
\end{tabular}

En las categorías de esta variable, en el GE 15 (100\%) mostraron CAC deficientes durante el pre test, logrando en el pos test que el 100\% se ubicara con CAC especializadas; por lo que es la primera muestra que la intervención de enfermería diseñada logró mejorar las CAC (Ver Tabla 4).

Tabla 4. Categorías de las Capacidades de Autocuidado

\begin{tabular}{|c|c|c|c|c|c|c|c|c|}
\hline \multirow[t]{3}{*}{ CAC } & \multicolumn{4}{|c|}{ Grupo Control } & \multicolumn{4}{|c|}{ Grupo Experimental } \\
\hline & \multicolumn{2}{|c|}{$\mathrm{O}_{1}$} & \multicolumn{2}{|c|}{$\mathrm{O}_{2}$} & \multicolumn{2}{|c|}{$\mathrm{O}_{1}$} & \multicolumn{2}{|c|}{$\mathrm{O}_{2}$} \\
\hline & $f$ & $\%$ & $f$ & $\%$ & $f$ & $\%$ & $f$ & $\%$ \\
\hline Deficientes & 14 & 93.3 & 4 & 26.7 & 15 & 100 & 0 & 0 \\
\hline Regulares & 1 & 6.7 & 11 & 73.3 & 0 & 0 & 0 & 0 \\
\hline Especializadas & 0 & 0 & 0 & 0 & 0 & 0 & 15 & 100 \\
\hline Total & 15 & 100 & 15 & 100 & 15 & 100 & 15 & 100 \\
\hline
\end{tabular}

Para dar cumplimento al Objetivo Especifico No. 2 donde se pretende identificar el nivel de ES en las personas con DM2 se realiza la categorización de los 7 factores seleccionados de acuerdo a la NOM-015-SSA2-2010 para la atención, tratamiento y control de la DM; como se muestra en el cuadro el GE se mostraron cifras con tendencia a la disminución en 6 (Glucemia Capilar, Hemoglobina Glucosilada, Colesterol, Triglicéridos, TAD y TAS) de los 7 factores; indicando que el único que mostró mínimas diferencias entre el pre y post prueba fue el IMC. 
Tabla 5. Categorías de los factores que integran el Estado de Salud

\begin{tabular}{|c|c|c|c|c|c|c|c|c|c|}
\hline \multirow{2}{*}{\multicolumn{2}{|c|}{ Estado de Salud }} & \multicolumn{4}{|c|}{ Grupo Control } & \multicolumn{4}{|c|}{ Grupo Experimental } \\
\hline & & \multicolumn{2}{|c|}{$\mathrm{O}_{1}$} & \multicolumn{2}{|c|}{$\mathrm{O}_{2}$} & \multicolumn{2}{|c|}{$\mathrm{O}_{1}$} & \multicolumn{2}{|c|}{$\mathrm{O}_{2}$} \\
\hline & & $f$ & $\%$ & $f$ & $\%$ & $f$ & $\%$ & $f$ & $\%$ \\
\hline \multirow[t]{3}{*}{ ÍMC } & Bueno & 1 & 6.7 & 0 & 0 & 0 & 0 & 1 & 6.7 \\
\hline & Regular & 0 & 0 & 1 & 6.7 & 3 & 20 & 5 & 33.3 \\
\hline & Malo & 14 & 93.3 & 14 & 93.3 & 12 & 80 & 9 & 60 \\
\hline \multirow[t]{2}{*}{ TAD } & Bueno & 7 & 46.7 & 6 & 40 & 7 & 46.7 & 15 & 100 \\
\hline & Malo & 8 & 53.3 & 9 & 60 & 8 & 53.3 & 0 & 0 \\
\hline \multirow[t]{3}{*}{ TAS } & Bueno & 7 & 46.7 & 6 & 40 & 10 & 66.7 & 14 & 93.3 \\
\hline & Regular & 2 & 13.3 & 0 & 0 & 1 & 6.7 & 1 & 6.7 \\
\hline & Malo & 6 & 40 & 9 & 60 & 4 & 26.7 & 0 & 0 \\
\hline \multirow[t]{3}{*}{ Colesterol } & Bueno & 7 & 46.7 & 7 & 46.7 & 0 & 0 & 7 & 46.7 \\
\hline & Regular & 5 & 33.3 & 6 & 40 & 7 & 46.7 & 4 & 26.7 \\
\hline & Malo & 3 & 20 & 2 & 13.3 & 8 & 53.3 & 4 & 26.7 \\
\hline \multirow[t]{3}{*}{ Triglicéridos } & Bueno & 0 & 0 & 1 & 6.7 & 1 & 6.7 & 15 & 100 \\
\hline & Regular & 11 & 73.3 & 5 & 33.3 & 10 & 66.7 & 0 & 0 \\
\hline & Malo & 4 & 26.7 & 9 & 60 & 4 & 26.7 & 0 & 0 \\
\hline Glicemia & Bueno & 0 & 0 & 0 & 0 & 0 & 0 & 13 & 86.7 \\
\hline \multirow[t]{2}{*}{ Capilar } & Regular & 1 & 6.7 & 0 & 0 & 0 & 0 & 2 & 13.3 \\
\hline & Malo & 14 & 93.3 & 15 & 100 & 15 & 0 & 0 & 0 \\
\hline Hemoglobina & Bueno & 0 & 0 & 2 & 13.3 & 0 & 0 & 13 & 86.7 \\
\hline \multirow[t]{2}{*}{ Glucosilada } & Regular & 12 & 80 & 11 & 73.3 & 9 & 60 & 2 & 13.3 \\
\hline & Malo & 3 & 20 & 2 & 13.3 & 6 & 40 & 0 & 0 \\
\hline
\end{tabular}

Para dar cumplimiento al Objetivo Especifico No. 3 en la identificación de la relación de las variables clínicas que corresponden al ES y las CAC se realiza la estadística de prueba de normalidad Smirnov y Kolmogorov con una intervalo de confianza del 95\%, se elige esta prueba debido a que se utiliza en grupos mayores de 30 sujetos con el objetivo de medir el grado de concordancia existente entre la distribución de un conjunto de datos y una distribución teórica especifica que permite elegir el coeficiente de correlación a utilizar en lo que se obtiene el rechazo de la hipótesis nula $[p<\alpha(0.05)]$; por lo que se elige el coeficiente de correlación de Pearson.

Como se observa en la matriz de correlación de Pearson; las CAC presentan relación negativa significativa con el IMC [ $\mathrm{rs}=-.330(\mathrm{p}<0.01)]$, glicemia capilar $[\mathrm{rs}=-.542 \quad(\mathrm{p}<0.01)]$, colesterol $[\mathrm{rs}=-.367 \quad(\mathrm{p}<0.01)]$, triglicéridos $[\mathrm{rs}=-.587 \quad(\mathrm{p}<0.01)]$ y hemoglobina glucosilada [rs $=-.478$ $(\mathrm{p}<0.01)$ ]; lo que demuestra que de los componentes denominados FCB, las variables que integran el ES mantiene una relación negativa significativa con las CAC, no ocurriendo de esta forma con la edad (Ver Tabla 6). 
Tabla 6. Matriz de correlación de Pearson del GC y GE

\begin{tabular}{lrrrrrrr}
\hline & Edad & IMC & GC & C & T & HG & CAC \\
\hline Edad & 1.00 & & & & & & \\
IMC & .136 & 1.00 & & & & & \\
GC & -.053 & .083 & 1.00 & & & & \\
C & .088 & .218 & $\mathbf{. 2 6 0 *}$ & 1.00 & & & \\
T & .038 & $\mathbf{. 3 1 0} *$ & $\mathbf{. 3 6 3} * *$ & $\mathbf{. 3 0 7 *}$ & 1.00 & & \\
HG & -.061 & .052 & $\mathbf{. 9 8 1} * *$ & $\mathbf{. 3 1 0}$ & $\mathbf{. 2 8 7 *}$ & 1.00 & \\
CAC & -.025 & $\mathbf{- . 3 3 0 * *}$ & $\mathbf{. 5 4 2} * *$ & $\mathbf{- . 3 6 7 * *}$ & $\mathbf{- . 5 8 7 *} *$ & $\mathbf{- . 4 7 8 * *}$ & 1.00 \\
\hline
\end{tabular}

*La correlación es significativa en el nivel 0.05 ( 2 colas)

**La correlación es significativa en el nivel 0.01 ( 2 colas)

\section{Estadística Inferencial}

Para dar cumplimiento al Objetivo General se realiza la estadística de prueba de normalidad Shapiro Wilk debido a que esta se utiliza en grupos menores a 30 sujetos con el objetivo de medir el grado de concordancia existente entre la distribución de un conjunto de datos y una distribución teórica especifica que permite elegir la estadística de prueba de hipótesis a utilizar, en lo que se obtiene el rechazo de la hipótesis nula $(p>\alpha)$ con los siguientes resultados: en el GC durante el pre test obtuvo una $p=.318(\alpha=0.05)$, $y$ en el pos test $p=.386(\alpha=0.05)$; mientras que el GE en el pre test obtuvo una $\mathrm{p}=.076(\alpha=0.05)$ y en el pos test $\mathrm{p}=.334(\alpha=0.05)$; por lo que se elige la prueba $\mathrm{T}$ de Student para muestran relacionadas y para muestras independientes (Ver Tabla 7).

Tabla 7. Prueba de Normalidad de Shapiro-Wilk

\begin{tabular}{lccc}
\hline & \multicolumn{3}{c}{ Shapiro-Wilk } \\
\cline { 2 - 4 } \multicolumn{1}{c}{ Grupos participantes de la IE } & Estadístico & gl & Sig. \\
\hline GC (Pre test) & .934 & 15 & .318 \\
GC (Pos Test) & .939 & 15 & .368 \\
GE (Pre test) & .893 & 15 & .076 \\
GE (Pos test) & .936 & 15 & .334 \\
\hline
\end{tabular}

Seleccionada la estadística de prueba $\mathrm{T}$ de Student para muestras independientes con la finalidad de establecer la igualdad de varianzas entre las mediciones realizadas en el GC y GE en el pre test. En lo correspondiente a la primera medición o pre test se asumen la igualdad de varianza con un intervalo de confianza del 95\%; obteniendo como resultado una $\mathrm{t}=2.186(\mathrm{gl}=28)$ $(\mathrm{p}<0.05)$, por lo que se concluye que no existe diferencia significativa entre la pre prueba en ambos grupos, lo anterior refleja que no existen diferencias entre el GC y GE al inicio de la intervención, estableciendo que los participantes del estudio mantienen características similares (Ver Tabla 8).

Tabla 8. Prueba T de Student para muestras independientes (GC y GE Pre test)

Variable

Prueba de

Levene

Prueba t para la igualdad de medias 
Sig. Dif. de Dif. $95 \%$ de IC

F Sig. $\quad \mathrm{T}$ Gl (bilateral) medias $\mathrm{EE}$ Inf.

CAC Se

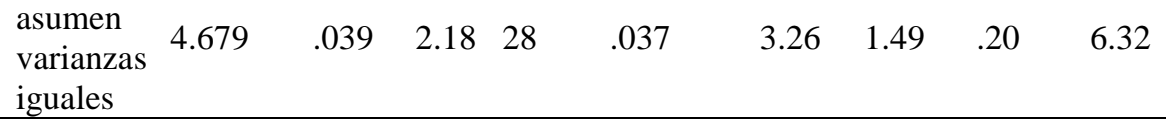

Al realizar el análisis estadístico en la pos prueba en el GC y GE obtiene una $\mathrm{t}=-19.144(\mathrm{gl}=28)(\mathrm{p}>0.05)$ con un $95 \%$ de intervalo de confianza de la diferencia, asumiendo que existe diferencia estadística entre el GC y GE encontrándose una diferencia de medias de -29.06667 (Ver Tabla 9).

Tabla 9. Prueba T de Student para muestras independientes (GC y GE Pos test)

\section{Prueba de}

Levene Prueba t para la igualdad de medias

Dif. $\quad 95 \%$ de IC de la

\begin{tabular}{|c|c|c|c|c|c|c|c|c|c|c|}
\hline \multirow{2}{*}{\multicolumn{2}{|c|}{ Variable }} & \multirow[b]{2}{*}{$\mathrm{F}$} & \multirow[b]{2}{*}{ Sig. } & \multirow[b]{2}{*}{$\mathrm{t}$} & \multirow[b]{2}{*}{ Gl } & \multirow{2}{*}{$\begin{array}{c}\text { Sig. } \\
\text { (bilateral) }\end{array}$} & \multirow{2}{*}{$\begin{array}{l}\text { Dif. de } \\
\text { medias }\end{array}$} & \multirow{2}{*}{$\begin{array}{l}\text { de } \\
\text { EE }\end{array}$} & \multicolumn{2}{|c|}{ Dif. } \\
\hline & & & & & & & & & Inf. & Sup. \\
\hline CAC & $\begin{array}{l}\text { Se } \\
\text { asumen } \\
\text { varianzas } \\
\text { iguales }\end{array}$ & 4.154 & .05 & -19.14 & 28 & .000 & -29.06 & 1.51 & -32.17 & -25.95 \\
\hline
\end{tabular}

En lo referente al efecto de la IEE en las CAC, los resultados muestran una $r s=.527(\mathrm{p}=0.043)$; lo que significa que al realizar la prueba $\mathrm{T}$ de Student para muestras relacionadas se obtuvo una $\mathrm{t}=-61.588$ con 14 grados de libertad para un intervalo de confianza del $95 \%$, ubicando una media de -42.86667 (Ver Tabla 10 y 11).; lo que muestra que existe una diferencia de medias entra la $\mathrm{O}_{1}$ con una media de 39.333 y la $\mathrm{O}_{2}$ con una media de 82.2 (Ver Tabla 3); por lo que base a estos resultados existe una diferencia estadística entre el pre y pos test del GE, mostrando que la IEE aumenta las CAC.

Tabla 10. Correlaciones de muestras emparejadas

\begin{tabular}{lrrr}
\hline Grupo Experimental & $n$ & Correlación & Sig. \\
\hline $\mathrm{O}_{1}-\mathrm{O}_{2}$ & 15 & .527 & .043 \\
\hline
\end{tabular}

Tabla 11. Prueba T de Student para muestras relacionadas (GE Pre y Pos test)

\begin{tabular}{|c|c|c|c|c|c|c|c|c|}
\hline \multirow[b]{3}{*}{ CAC } & \multicolumn{5}{|c|}{ Diferencias emparejadas } & \multirow[b]{3}{*}{$\mathrm{T}$} & \multirow[b]{3}{*}{ gl } & \multirow{3}{*}{$\begin{array}{c}\text { Sig. } \\
\text { (bilateral) }\end{array}$} \\
\hline & \multirow[b]{2}{*}{ Media } & \multirow[b]{2}{*}{$\mathrm{DE}$} & \multirow[b]{2}{*}{$X$ de $\mathrm{EE}$} & \multicolumn{2}{|c|}{$\begin{array}{c}95 \% \text { de IC de la } \\
\text { Dif. }\end{array}$} & & & \\
\hline & & & & Inferior & Superior & & & \\
\hline $\mathrm{O}_{1}-\mathrm{O}_{2}$ & -42.86 & 2.69 & .69 & -44.35 & -41.37 & -61.58 & 14 & .000 \\
\hline
\end{tabular}

De igual manera el efecto de la IEE en las variables clínicas que corresponden al ES muestran que el IMC ( $\mathrm{t}=13.926)$, TAD $(\mathrm{t}=2.843)$, TAS $(\mathrm{t}=2.628)$, Glicemia Capilar $(\mathrm{t}=7.658)$, Colesterol $(\mathrm{t}=4.685)$, Triglicéridos 
$(\mathrm{t}=9.363)$ y Hemoglobina Glucosilada $(\mathrm{t}=5.118)$ con 14 grados de libertad para un intervalo de confianza del 95\%; obteniendo una media: Índice de Masa Corporal (1.373), Tensión Arterial Diastólica (10.3), Tensión Arterial Sistólica (7.0), Glicemia Capilar (92.533), Colesterol (37.267), Triglicéridos (45.067) y Hemoglobina Glucosilada (2.10667) (Ver Tabla 12 y 13).

Tabla 12. Correlaciones de muestras emparejadas

\begin{tabular}{lrrr}
\hline $\begin{array}{l}\text { Estado de Salud } \\
\left(\mathrm{O}_{1}-\mathrm{O}_{2}\right)\end{array}$ & $n$ & Correlación & Sig. \\
\hline Índice de Masa Corporal & & .527 & .043 \\
Tensión Arterial Diastólica & & .993 & .000 \\
Tensión Arterial Sistólica & 15 & -.023 & .936 \\
Glicemia Capilar & & .487 & .065 \\
Colesterol & & .727 & .002 \\
Triglicéridos & .809 & .000 \\
Hemoglobina Glucosilada & .651 & .009 \\
\hline
\end{tabular}

Tabla 13. Prueba T de Student para muestras relacionadas (GE Pre y Pos test)

\begin{tabular}{|c|c|c|c|c|c|c|c|c|}
\hline \multirow{3}{*}{$\begin{array}{l}\text { Estado de Salud } \\
\left(\mathrm{O}_{1}-\mathrm{O}_{2}\right)\end{array}$} & \multicolumn{5}{|c|}{ Diferencias emparejadas } & \multirow[b]{3}{*}{$\mathrm{t}$} & \multirow[b]{3}{*}{ Gl } & \multirow{3}{*}{$\begin{array}{c}\text { Sig. } \\
\text { (bilateral) }\end{array}$} \\
\hline & \multirow[b]{2}{*}{ Media } & \multirow[b]{2}{*}{ DE } & \multirow{2}{*}{$\begin{array}{r}\mathrm{X} \text { de } \\
\mathrm{EE}\end{array}$} & \multicolumn{2}{|c|}{$\begin{array}{c}95 \% \text { de IC de la } \\
\text { Dif. }\end{array}$} & & & \\
\hline & & & & Inferior & Superior & & & \\
\hline $\begin{array}{l}\text { Índice de Masa } \\
\text { Corporal }\end{array}$ & 1.37 & .38 & .09 & 1.16 & 1.58 & 13.92 & 14 & .000 \\
\hline $\begin{array}{l}\text { Tensión Arterial } \\
\text { Diastólica }\end{array}$ & 10.33 & 14.07 & 3.63 & 2.53 & 18.12 & 2.84 & 14 & .013 \\
\hline $\begin{array}{l}\text { Tensión Arterial } \\
\text { Sistólica }\end{array}$ & 7.00 & 10.31 & 2.66 & 1.28 & 12.71 & 2.62 & 14 & .020 \\
\hline Glicemia Capilar & 92.53 & 46.80 & 12.08 & 66.61 & 118.45 & 7.65 & 14 & .000 \\
\hline Colesterol & 37.26 & 30.80 & 7.95 & 20.20 & 54.32 & 4.68 & 14 & .000 \\
\hline Triglicéridos & 45.06 & 18.64 & 4.81 & 34.74 & 55.39 & 9.36 & 14 & .000 \\
\hline $\begin{array}{l}\text { Hemoglobina } \\
\text { Glucosilada }\end{array}$ & 2.10 & 1.59 & .41 & 1.22 & 2.98 & 5.11 & 14 & .000 \\
\hline
\end{tabular}

Dado que las medias en la pre y pos pruebas oscilaron: IMC (29.7928.42), TAD (84-73.67), TAS (120-113), Glicemia Capilar (217.13-124.6), Colesterol (213.73-176.47), Triglicéridos (185.93-140.87), y Hemoglobina Glucosilada (8.31-6.20) respectivamente; en base a lo anterior se encuentran diferencias estadísticas de medias entre el pre y pos test del GE en las ocho principales variables clínicas que integran el ES, haciendo referencia que la IEE mejora el ES (Ver Tabla 5).

\section{Discusión}

En los resultados del nivel de CAC este estudio muestra disparidades con los reportados en investigaciones previas; por ejemplo, Contreras, Contreras-Manchado, Hernández, Castro y Navarro (2013) encontraron que el 
88.89\% de los diabéticos tiene CAC regulares y el estudio de Romero, Dos Santos, Aparecida y Zanetti (2010) ubicó que 168 (66.5\%) mostraron CAC regulares. De igual manera Herrera, Andrade, Manrique y Mayerli (2012) encontró que el $73.8 \%$ de los diabéticos obtuvo muy buena CAC, que coincide con el reporte de Cadena y Flores (2010) donde el $67 \%$ reflejó buena CAC; sin embargo, este estudio mostró que 29 (96.6\%) reflejaron CAC deficientes en el pre test y $50 \%$ mostró CAC especializadas en el pos test.

En lo referente a la relación de las variables sociodemográficas, variables clínicas que conforman el ES y las CAC este estudio muestra diferencias con lo reportado por Romero, Dos Santos, Aparecida y Zanetti (2010) donde ubicaron una correlación directamente proporcional entre CAC y años de estudio $(r=0.124 ; \mathrm{p}<0.05)$ y una correlación negativa entre las CAC y el tiempo de evolución de la enfermedad ( $\mathrm{r}=-0.667$; $\mathrm{p}<0.05)$; sin embargo, este estudio mostró que las CAC presentan relación negativa significativa con el IMC [rs=-.330 $(\mathrm{p}<0.01)]$, tensión arterial diastólica $[\mathrm{rs}=-.441(\mathrm{p}<0.01)]$, tensión arterial sistólica $[\mathrm{rs}=-.350(\mathrm{p}<0.01)]$, glicemia capilar $[\mathrm{rs}=-.542$ $(\mathrm{p}<0.01)]$, colesterol [rs=-.367 $(\mathrm{p}<0.01)]$, triglicéridos $[\mathrm{rs}=-.587(\mathrm{p}<0.01)]$ y hemoglobina glucosilada [rs=-.478 $(\mathrm{p}<0.01)]$; no ocurriendo de esta forma con la edad y el tiempo de diagnóstico.

En cuanto a los resultados de la IEE este estudio reporta semejanzas con el realizado por Maya, Hernández y Luna (2008) en el Distrito Federal donde con una $n=38$ pacientes divididos en un GC y GC realizaron una intervención, encontrando en el GE un incremento de las CAC especializadas de $69.47 \%$ a $82.33 \%$; además de otros indicadores que registraron disminución: Glucemia Capilar y el IMC.

De igual manera se coincide con lo reportado por Ávila, Meza, Frías, Sánchez, Vega y Hernández (2006) donde aplicaron una intervención de enfermería en la CAC de las personas con DM2 utilizando el sistema de apoyo educativo; teniendo una $n=31$ personas con DM2; a través de una prueba $\mathrm{t}$ pareada se observó que existe diferencia significativa en la pre prueba y la pos prueba de la intervención de enfermería con una significancia estadística en la CAC después de recibir apoyo educativo de enfermería [ $\mathrm{t}=3.579 \mathrm{p}=002]$.

En este estudio se tuvo una muestra de 30 sujetos; al realizar la prueba $\mathrm{T}$ de Student para muestras independientes con la finalidad de establecer la igualdad de varianzas entre las mediciones realizadas en el GC y GE en el pre test; los resultados asumen la igualdad de varianzas con un intervalo de confianza del 95\%; obteniendo como resultado $[\mathrm{t}=2.186(\mathrm{gl}=28)(\mathrm{p}<0.05)]$, por lo que se concluye que no existe diferencia significativa entre la pre prueba en ambos grupos, teniendo por lo tanto características similares.

El análisis estadístico en la pos prueba en el GC y GE a través de una prueba $\mathrm{T}$ de Student para muestras independientes $[\mathrm{t}=-19.144 \quad(\mathrm{gl}=28)$ ( $\mathrm{p}>0.05)$ ] con un $95 \%$ de intervalo de confianza de la diferencia, asumiendo 
que existe diferencia estadística en el GC y GE encontrándose una diferencia de medias de -29.06667 en el pos test.

En lo referente al efecto de la IEE en las CAC, los resultados muestran una $\mathrm{rs}=.527(\mathrm{p}=0.043)$; al realizar la prueba $\mathrm{T}$ de Student para muestras relacionadas se obtuvo una $\mathrm{t}=-61.588$ con 14 grados de libertad para un intervalo de confianza del 95\%, ubicando una media de -42.86667 (Ver Tabla 12 y 13); lo que muestra que existe una diferencia de medias entra la $\mathrm{O}_{1}$ con una media de 39.333 y la $\mathrm{O}_{2}$ con una media de 82.2 (Ver Tabla 5); en base a estos resultados existe una diferencia estadística entre el pre y pos test del GE, mostrando que la IEE aumenta las CAC.

\section{Conclusión}

La DM2 es una enfermedad crónica degenerativa multifactorial que implica en la persona la adopción de medidas que favorezcan el autocuidado; por lo que es necesario que el diabético muestre CAC que culmine en AC; y por lo tanto mantenga un equilibrio de los Factores Condicionantes Básicos y los requisitos de autocuidado universales, por desviación de salud y del desarrollo que tengan como resultado final el aumento en la Esperanza de Vida y la Calidad de Vida. El papel del profesional de enfermería en el primer nivel de atención a la salud es de suma importancia; pues requiere el diseño de intervenciones educativas de enfermería que incrementen la CAC y mejore el ES de las personas con DM2.

Las CAC mostraron que casi en su totalidad (96.6\%) se categorizan en deficientes; además se encontraron relaciones negativas significativas entre la CAC y el peso, IMC, TAD, TAS, glucemia capilar, colesterol, triglicéridos y hemoglobina glucosilada; no ocurriendo de esta forma con la edad y años de diagnóstico. Se demostró un efecto en los participantes del GE con el aumento de las CAC y la disminución de las cifras en las variables clínicas que integraron el ES (Peso, Estatura, IMC, Colesterol, Triglicéridos, Glucemia Capilar y Hemoglobina Glucosilada) lo que explica efecto de la IEE en las CAC y mejora del ES de las personas con DM2.

\section{References:}

1. Organización Mundial de la Salud (2016). Informe mundial sobre la diabetes.

2. Instituto Nacional de Salud Pública. Encuesta Nacional de Salud y Nutrición de Medio Camino 2016 Informe Final de Resultados.

3. Ávila A. H., Meza G. S., Frías R. B., Sánchez A. E., Vega A. C. \& Hernández S. Ma . A. (2006). Intervención de enfermería en el autocuidado con apoyo educativo en personas con DM2. Revista cultura de los cuidados, 20 (10): 141-146. 
4. Bulechek G. M., Butcher H. K., Dochterman J. M. \& Wagner C. M. (2013). Clasificación de Intervenciones de Enfermeria (NIC). 6ta. Edición. Elsevier, España.

5. Contreras O. A., Contreras M. A., Hernández B. C., Castro B. MT. \& Navarro P. LL. (2013). Capacidad de autocuidado en pacientes diabéticos que asisten a consulta externa. Sincelejo, Colombia. Investigaciones Andinas; 15 (26): 667-678.

6. Córdova V., J. Á., et. al. (2008). Las enfermedades crónicas no transmisibles en México: sinopsis epidemiológica y prevención integral. Revista de Salud Pública México; 50:419-427

7. Cutcliffe J., Mckenna H. \& Hyrkas C. (2011). Modelos de Enfermería: aplicación a la práctica. (2da. Edición) México, D.F.; Manual Moderno.

8. Fernández A. R. (2010). Efecto de una intervención educativa de enfermería en el fortalecimiento de la capacidad de agencia de autocuidado del adulto mayor hipertenso ambulatorio de Tunjaboyacá, Colombia. Tesis doctoral, Universidad Nacional de Colombia.

9. Hernández S. R., Fernández C. C \& Baptista L. Pilar (2014). Metodología de la investigación, 6ta. Edición, Mc Graw Hill: 220-265.

10. Herrera L. A., Yesid R., Andrade H., Orlando H. S., Manrique M. JP., Faria C. KL \& Machado R. M. (2012). Personas con diabetes mellitus tipo 2 y su capacidad de agencia de autocuidado, Cartagena. Revista Avances en Enfermería; 30 (2): 39-46.

11. Instituto Nacional de Estadística Española (2013). Encuesta Nacional de Salud Española (ENSE) 2011-2012. Ministerio de Sanidad, Servicios Sociales e Igualdad; Notas de Prensa: 1-12.

12. Instituto Nacional de Salud Pública (2012). Encuesta Nacional de Salud y Nutrición. Secretaría de Salud; 19-23.

13. Kozier B., Berman, A., Snyder S. J. \& Erb G. (2013). Teorías de Enfermería y marcos conceptuales. Fundamentos de Enfermería. Editorial Pearson 9a ed., Madrid, España; 44.

14. Landeros O. EA. \& Gallegos C. E. (2005). Capacidades de autocuidado y percepción del estado de salud en adultos con y sin obesidad. Tesis de posgrado Maestría en Ciencias de Enfermería, Universidad Autónoma de Nuevo León.

15. Lezama J., Malavé C. \& Tovar M. (2005). Programa de orientación sobre autocuidado a los usuarios con diabetes tipo 2 que asisten a la unidad de diabetes Dra. Iris García de Mota del hospital Dr. Julio Rodríguez de Cumana - estado Sucre. Tesis de grado, Universidad Central de Venezuela. 
16. Maya A., Hernández J. \& Luna J. (2008). Capacidades especializadas de autocuidado y adaptación de los pacientes con diabetes mellitus tipo 2. Revista CONAMED; 13: 30-35.

17. Orem D. (1993). Modelo de Orem: conceptos de enfermería en la práctica. 1ra. Edición, Editorial Masson.

18. Organización Mundial de la Salud (2010). Vigilancia de las enfermedades no transmisibles (ENT).

19. Organización Mundial de la Salud (2010). La diabetes es una enfermedad crónica.

20. Organización Mundial de la Salud (2013). Nota descriptiva No. 312.

21. Organización Mundial de la Salud (2013). 10 datos sobre la Diabetes: datos y cifras.

22. Organización Panamericana de la Salud. (2010). Estadísticas vitales de salud.

23. Polit, D. F. \& Hungler, B. P. (2000). Investigación científica en ciencias de la salud. (6 ${ }^{\mathrm{a}}$ ed.) México, D.F.: McGraw-Hill Interamericana.

24. Raile A. M (2014). Modelos y Teorías de Enfermeria. 8va. Edición Elsevier, España.

25. Rodríguez G. MÁ., Arredondo H. E. \& Salamanca A. YA. (2013). Capacidad de agencia de autocuidado y factores relacionados con la agencia en personas con insuficiencia cardíaca de la ciudad de Medellín (Colombia). Enfermería Global; 30: 183-195.

26. Rodríguez S. \& Vallenilla C. (2007). Intervención educativa de la enfermera comunitaria en la promoción del autocuidado en el paciente diabético atendido en la unidad diabetológica del hospital distrital de bejuma. Estado Carabobo. Tesis de grado, Universidad Central de Venezuela.

27. Romero B. I., Dos Santos MA., Aparecida M. T. \& Zanetti ML. (2010). Autocuidado de personas con Diabetes Mellitus atendidas en un servicio de urgencia en México. Revista Latinoamericana de Enfermagem, 18 (6): 1-9.

28. Secretaría de Salud (2013). Enfermedades metabólicas. Programa Nacional de Salud (2013-2018); Diario Oficial de la Federación.

29. Secretaría de Salud (2010). NOM-015-SSA2-2010, Para la prevención, tratamiento y control de la diabetes mellitus en la atención primaria; Diario Oficial de la Federación.

30. Secretaría de Salud (1984). Ley General de Salud. Leyes y Códigos de México. (16 ${ }^{\mathrm{a}}$. Ed.) Reglamento de la Ley General de Salud en Materia de Investigación para la Salud. México: Porrúa. 